\title{
Effect of grain boundaries on hydrocarbon sensing in Fe-doped $p$-type semiconducting perovskite $\mathrm{SrTiO}_{3}$ films
}

\author{
M. Mahesh Kumar ${ }^{\mathrm{a})}$ and M. L. Post \\ Institute for Chemical Process and Environmental Technology, National Research Council of Canada, \\ 1200 Montreal Road, Ottawa, Ontario KIA OR6, Canada
}

(Received 1 February 2005; accepted 5 April 2005; published online 7 June 2005)

\begin{abstract}
Films of Fe-doped $\mathrm{SrTiO}_{3}$ deposited using pulsed laser deposition on sapphire and alumina, when exposed to propane, showed different sensor responses measured (as a resistance change) as a function of temperature and microstructure. The film deposited on alumina has a stronger response towards 3000-ppm propane than does the film deposited on sapphire. Films deposited on alumina exhibit higher dc conductivity than the films on sapphire. The activation energies indicate a mixed electronic/ionic conduction at low temperature with the high-temperature regime showing a temperature-independent conductivity. The origin of the difference in gas responses caused by varying temperature and morphology has been explored using ac impedance techniques and measured as a function of frequency $(1 \mathrm{~Hz} \leqslant f \leqslant 1 \mathrm{MHz})$ and temperature $\left(200 \leqslant T \leqslant 480{ }^{\circ} \mathrm{C}\right)$. A single relaxation (a single semicircle in the complex impedance plane) in the frequency domain was observed in addition to the relaxation due to the electrode-film interface. A model and a mechanism of conduction for the above are derived using equivalent circuits to fit the ac impedance data and dc conductivity. It is proposed that the reduction of Fe-doped $\mathrm{SrTiO}_{3}$, which is induced by propane, enhances the space-charge barrier near the grain boundaries and increases the sensitivity to propane. (c) 2005 American Institute of Physics. [DOI: 10.1063/1.1922584]
\end{abstract}

\section{INTRODUCTION}

Demand for chemical sensors has been growing at a consistent pace in recent years due to the stringent environmental regulations that are coming into effect to reduce emissions and hazardous pollutants. Miniaturization and severe environmental conditions have set demands on such sensors. Ideal sensors should not only selectively and sensitively measure the presence of one particular gas but also must be robust and cost effective. Several metal oxide sensors which operate on the principle of conductometric changes have been in the market for a while, to sense gases ranging from simple oxygen to complex hydrocarbons. The most commonly available are based on doped $\mathrm{SnO}_{2}$ and $\mathrm{ZnO}{ }^{1}$

One feature that is common to most of the commercially available conductometric sensors is that the conductivity is of $n$ type and there are only a few sensors which have $p$-type conductivity. The metal oxide $\mathrm{SnO}_{2}$ is one of the wellinvestigated and better understood semiconductor gas sensors that has been widely used to detect low concentrations of gases. However, $\mathrm{SnO}_{2}$ sensors suffer from the poor selectivity and low hydrothermal stability necessitating a more selective and stable sensor. ${ }^{2-4}$ Another important factor to consider while performing a sensor measurement (which is generally resistance as a function of time and gas concentration) in an $n$-type sensor such as $\mathrm{SnO}_{2}$ is the magnitude of the resistance. In an $n$-type semiconductor, the resistance decreases in the presence of a reducing gas and should there be a large resistance decrease, the conventional two-probe resistivity measurements become untenable at low values of re-

\footnotetext{
a) Author to whom correspondence should be addressed; electronic mail: mahesh.matam@nrc.ca
}

sistance, compelling a change in the measurement technique and rendering it to misrepresentation of the true sensitivity.

As an alternative, a $p$-type semiconductor where the magnitude of resistance increases as a function of time in reducing gas concentrations has been chosen. The resistance rise allows sensor response measurement with no concerns about loss of accuracy at higher concentration of analytes. In addition, by modifying composition and morphology, the $p$-type semiconductors can be made stable for fluctuating temperatures and humidity. Materials with perovskite structure are receiving increased attention in this regard, which include simple perovskites such as $\mathrm{SrTiO}_{3}$ that are stable over a large temperature range. Similarly, $\mathrm{SrFeO}_{3-\delta}$ is another $p$-type semiconducting perovskite material, which has promising characteristics for a gas sensor. ${ }^{5}$ Unlike the former, the oxygen nonstoichiometry induces structural phase transitions in $\mathrm{SrFeO}_{3-\delta}(0<\delta<0.5)$. A change in oxygen stoichiometry leads to the redox reaction of $\mathrm{Fe}^{4+} \leftrightarrows \mathrm{Fe}^{3+}$ and the electronic transition constitutes a conduction mechanism which has repercussions in its gas-sensing properties. When these two perovskite compounds are mixed in stoichiometric molar ratios, solid solutions are formed, which are intrinsically depleted in oxygen. With varying oxygen stoichiometry, it was found that $\mathrm{Sr}(\mathrm{FeTi}) \mathrm{O}_{3-\delta}$ can show almost temperature-independent conductivity in a large regime at high temperatures, which is ideal for a gas sensor. ${ }^{5}$

Thin or thick film of the material on a substrate is the most common configuration that is used for a device in gassensing measurements. $\mathrm{Sr}(\mathrm{FeTi}) \mathrm{O}_{3}$, when exposed to reducing species such as hydrocarbons at atmospheric pressures, behaves as a $p$-type semiconductor and the rise in resistance 
could be mechanistically related to the surface reaction and the grain-boundary conduction, which the present paper addresses.

The effect of morphology and microstructute on electrical conductivity is an important parameter in polycrystalline titanate ceramics as they control a large number of properties. Examples are positive temperature coefficient of resistance, ${ }^{6}$ nonlinear power-law dependence of voltagecurrent (varistor) characteristics, ${ }^{7}$ solid electrolytes, ${ }^{8}$ and others. Impedance spectroscopy is a powerful technique which can be used to characterize the electrical microstructures of such properties. It allows an unambiguous dissemination of the several regions a ceramic is made up of, namely, grains, grain boundaries, electrodes, etc. The paper reports a model for the sensitivity of $\mathrm{Sr}(\mathrm{FeTi}) \mathrm{O}_{3}$ to propane by means of analyzing the ac impedance data measured over a large frequency and temperature ranges in both air and propane. It reports the results obtained on conductometric gas sensor responses, dc conductivity, and ac impedance spectroscopy, and proposes a model for the sensitivity for one particular composition of $\mathrm{SrTi}_{0.6} \mathrm{Fe}_{0.4} \mathrm{O}_{3-\delta}$ with propane. The measurements were done with films deposited by pulsed laser deposition and a comparison of the results obtained for films deposited on single-crystalline sapphire and on polycrystalline alumina has been undertaken.

\section{EXPERIMENT}

\section{A. Film preparation}

Targets of $\mathrm{SrTi}_{0.6} \mathrm{Fe}_{0.4} \mathrm{O}_{3}$ (hereafter called STF40), for pulsed laser deposition (PLD), are prepared by solid-state reactions using stoichiometric amounts of $\mathrm{SrCO}_{3}, \mathrm{Fe}_{2} \mathrm{O}_{3}$, and $\mathrm{TiO}_{2}$. The powders were thoroughly mixed and were prefired in a tube furnace in flowing $\mathrm{Ar}$ at $1050{ }^{\circ} \mathrm{C}$ for $1500 \mathrm{~min}$. Prior to pressing the powder into a pellet, the powders were again mixed and sintered at $1150{ }^{\circ} \mathrm{C}$ for $1500 \mathrm{~min}$ in flowing $\mathrm{O}_{2}$. A final sintering was carried out on pellets at $1430{ }^{\circ} \mathrm{C}$ for $1500 \mathrm{~min}$ in flowing oxygen. The targets obtained were dense $(80 \%)$ and black in color. The target was then used in PLD to deposit thin films of STF40 on substrates of singlecrystalline sapphire (1 $1 \overline{1} 02)$ and polycrystalline alumina. The PLD conditions for deposition were wavelength of the excimer laser (KrF) (Lambda Physik, LPX305i) $\lambda=248 \mathrm{~nm}$, pulse frequency of $8 \mathrm{~Hz}$, and energy of $600 \mathrm{~mJ}$. Fluence at the target was estimated to be $1.6 \mathrm{~J} / \mathrm{cm}^{2}$. The depositions were carried out in an atmosphere of oxygen at a partial pressure of $13.3 \mathrm{~Pa}$ with the substrate temperature at $700{ }^{\circ} \mathrm{C}$. After the deposition, films were annealed at the same temperature with oxygen partial pressure elevated to $53.3 \mathrm{kPa}$ for $30 \mathrm{~min}$ and the temperature then lowered to ambient at a controlled rate. The thickness of the films deposited was estimated to be $\sim 200 \mathrm{~nm}$.

\section{B. Structural and microstructural characterizations}

Phase identification was done on the sapphire- and alumina-coated films using $\mathrm{x}$-ray diffraction, measured in the $20^{\circ} \leqslant 2 \theta \leqslant 70^{\circ}$ range at room temperature using $\mathrm{Cu} K_{\alpha}$ radiation (Bruker D8 diffractometer). Images of microstructure

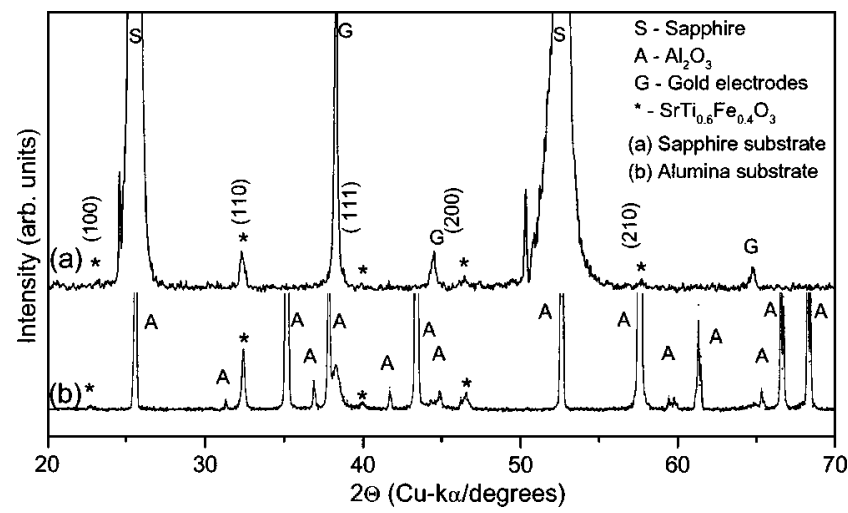

FIG. 1. X-ray diffraction patterns of the $\operatorname{SrTi}_{0.6} \mathrm{Fe}_{0.4} \mathrm{O}_{3}$ (STF40) deposited on (a) sapphire and (b) alumina. The unmarked intensities in (b) are from the substrate $\mathrm{Al}_{2} \mathrm{O}_{3}$ - and Au-evaporated electrodes. The asterisks (*) indicate the STF40 intensities.

were obtained using a field-emission gun scanning electron microscope (FEGSEM) of Hitachi S4800 with an accelerating voltage of $0.5 \mathrm{kV}$. Thermal analysis was measured on powder samples using a TA instruments' thermogravimetry analysis (TGA) in flowing air and in 3000-ppm propane after the temperature was stabilized at $450{ }^{\circ} \mathrm{C}$ for over $100 \mathrm{~min}$. Prior to this, the powders were taken to a temperature of $800{ }^{\circ} \mathrm{C}$ in air in order to remove volatiles and cooled to $450{ }^{\circ} \mathrm{C}$ at $10^{\circ} \mathrm{C} / \mathrm{min}$.

\section{Electrical characterization of films}

Prior to the electrical measurements, two gold pads were evaporated onto the film to serve as electrodes. Two-probe conductometric gas-sensing measurements in flowing air ( $21 \%$ oxygen in nitrogen) and propane (3000 ppm in synthetic air) were carried out in a 1-L chamber at a flow rate of $100 \mathrm{~cm}^{3} / \mathrm{min}$, where the temperature of the film could be varied between 30 and $500{ }^{\circ} \mathrm{C}$. dc conductivity and ac impedance studies were made using a Keithley 2400 DMM and a Solartron 1260 impedance analyzer, respectively, in the temperature range of $200 \leqslant T \leqslant 480^{\circ} \mathrm{C}$. dc conductivity was measured at $10{ }^{\circ} \mathrm{C}$ increments with temperature stabilization for over $30 \mathrm{~min}$. Impedance was measured at $20{ }^{\circ} \mathrm{C}$ intervals as function of frequency $(1 \mathrm{~Hz} \leqslant f \leqslant 1 \mathrm{MHz})$ in air and in propane. Impedance data were analyzed and fitted with equivalent circuits using the program ZVIEW 2.

\section{RESULTS AND DISCUSSION}

\section{A. X-ray diffraction}

It was found that $\mathrm{SrTiO}_{3}-\mathrm{SrFeO}_{3}$ forms a complete solid solution system with the structure distorting away from the cubic to orthorhombic to tetragonal with the increasing concentration of $\mathrm{SrFeO}_{3} .{ }^{9}$ This paper, however, reports the results obtained on the electrical properties of $60 \%$ $\mathrm{SrTiO}_{3}-40 \% \mathrm{SrFeO}_{3}(\mathrm{STF} 40)$ and has been selected in view of this film strongest response to propane. ${ }^{10}$

Figure 1 shows the x-ray diffraction pattern obtained for the STF40 films deposited on single-crystal sapphire and polycrystalline alumina at room temperature. Films deposited on sapphire show, in addition to the Bragg peaks from 

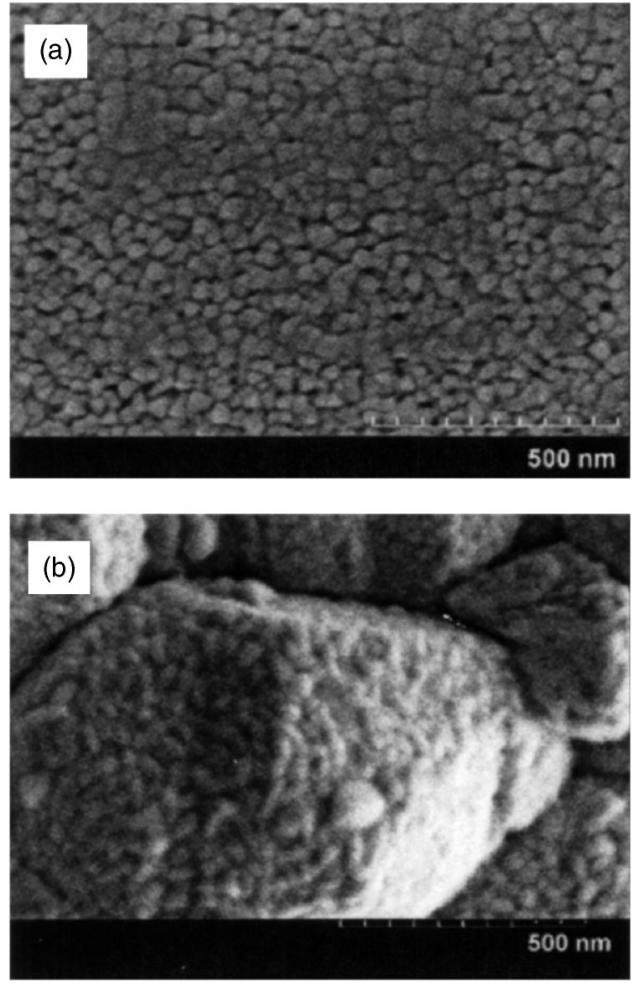

FIG. 2. Images of scanning electron microscope of $\mathrm{SrTi}_{0.6} \mathrm{Fe}_{0.4} \mathrm{O}_{3}$ on (a) sapphire and (b) alumina.

STF40, strong intensities of the sapphire substrate and the $\mathrm{Au}$ film used as the electrode. No impurity phase is evident and the structure is close to cubic perovskite, all the intensities of $\mathrm{SrTiO}_{3}$ are observed indicating the polycrystalline nature of the film. The $\mathrm{x}$-ray spectrum of the film deposited on polycrystalline alumina also shows a polycrystalline STF40 without traces of impurity.

\section{B. Microstructure}

The formation of a nanomorphology in the film using PLD is clearly revealed in the FEGSEM images shown in Fig. 2. The films of STF grown on sapphire [Fig. 2(a)] and alumina [Fig. 2(b)] show uniformity with grain sizes approximating to $\sim 50 \mathrm{~nm}$ [embedded nanograins of STF40 on the larger grains $(\sim 1 \mu \mathrm{m})$ of the substrate $\mathrm{Al}_{2} \mathrm{O}_{3}$ could be seen in Fig. 2(b)]. Films on sapphire appear to have a porous structure when compared to the film on alumina. A comparison of the films indicates that the film grown on alumina appears to have a greater density than the film grown on sapphire. The ensuing sections will show the profound effect that microstructure has on the propane-sensing characteristics of STF40. The sensor responses (detailed in the ensuing sections) reveal that the sensing characteristics are greatly affected by the substrate microstructure and the film thickness.

\section{Sensor response/dc conductometric measurements}

The conductometric sensor response of STF40 films was studied as a function of time and propane concentration. The response, measured as a change in dc resistance against time at various temperatures, is shown in Fig. 3. Figure 3(a)

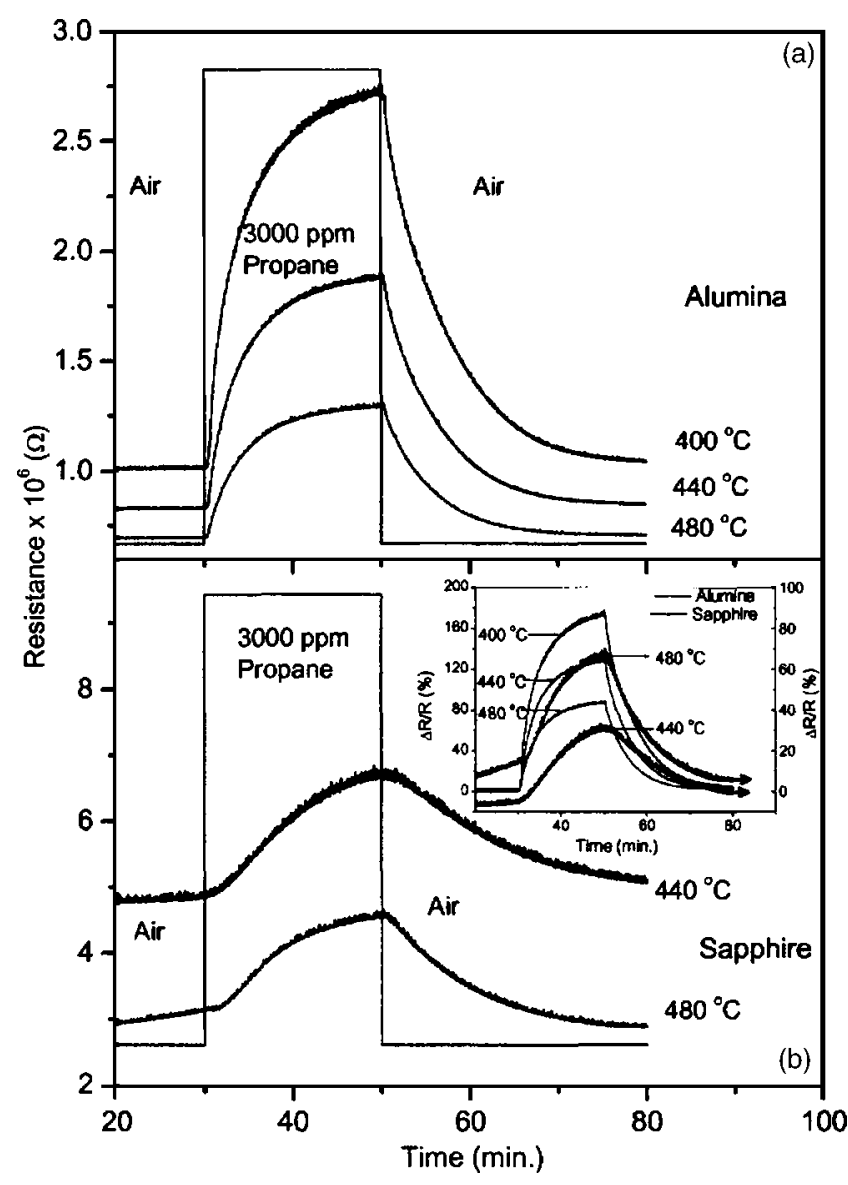

FIG. 3. Conductometric sensor responses in 3000-ppm propane and air. (a) $\mathrm{SrTi}_{0.6} \mathrm{Fe}_{0.4} \mathrm{O}_{3}$ film on alumina and (b) film on sapphire. Inset (b) relative response to propane calculated using Eq. (1).

shows the response of STF40 film deposited on alumina to 3000-ppm propane and Fig. 3(b) that of the film deposited on sapphire. Temperatures around $400{ }^{\circ} \mathrm{C}$ are optimized to provide adequately fast response kinetics to hydrocarbons. The percentage of response is calculated using the relation

$$
\frac{\Delta R}{R}(\%)=\frac{R_{\mathrm{gas}}-R_{0}}{R_{0}} \times 100 .
$$

The film deposited on alumina shows a stronger response [see inset of Fig. 3(b)] to 3000-ppm propane $(160 \%$ at $\left.400{ }^{\circ} \mathrm{C}\right)$ as compared to the film on sapphire $(65 \%$ at $\left.480{ }^{\circ} \mathrm{C}\right)$. In films deposited on sapphire not only that the response is smaller but also the response time is longer. This could be due to a slow reaction on the film surface due to higher density. However, the film on alumina reacts more rapidly producing a more rapid response. Another notable feature is that the STF40 film on alumina responds at a lower temperature to propane than STF40 on sapphire. The strongest response for the film deposited on alumina was obtained at $400{ }^{\circ} \mathrm{C}$ as opposed to $480{ }^{\circ} \mathrm{C}$ for the film on sapphire. Hence in all respects, the film on alumina appears to be a better choice for sensing a hydrocarbon such as propane than a film on sapphire. The influential parameter for the strong differences in responses may lie in the electrical microstructures of both the substrate that is used and film deposited on it. 

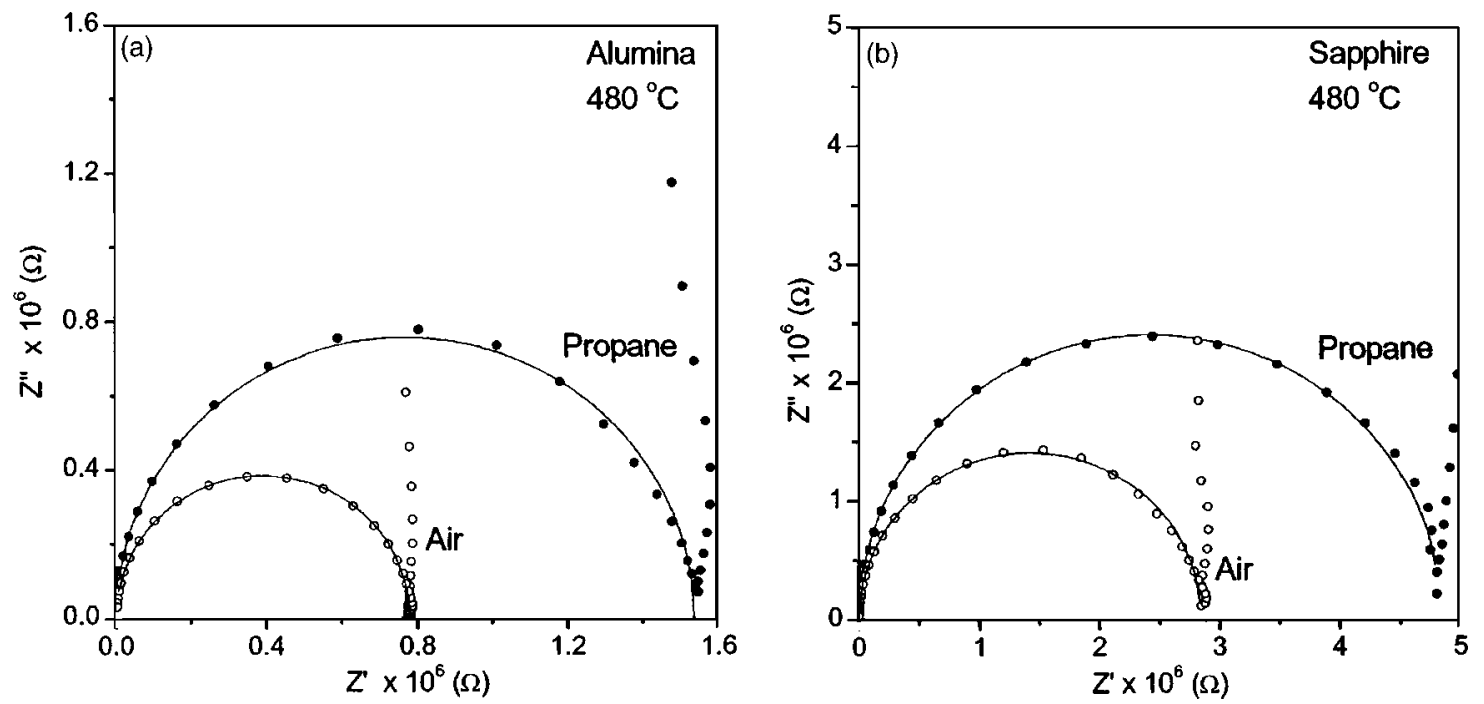

FIG. 4. Complex plane plots (Col-Col diagrams) for STF40 film deposited on (a) alumina and (b) sapphire. The lines are fits to the points using equivalent circuit model.

\section{Impedance spectroscopy}

As a member of the family of perovskite compounds, which show a variety of properties based on the microstructure, ${ }^{7,8}$ exploring electrical charge transport across the grains and grain boundaries is the key to understanding the hydrocarbon response to STF40. Strikingly, in $\mathrm{SrTiO}_{3}$ such as perovskites, it was found that the grain-boundary resistance dominates over the interiors of the bulk. ${ }^{11}$ Seminal works of Waser and co-workers ${ }^{12,13}$ have established through simulations that the grain-boundary potential barrier height and the conductivity near the space-charge depletion region increase the resistance greatly in an acceptor-doped $\mathrm{SrTiO}_{3}$. The enhanced response in the STF40 to propane is a result of the enhanced activity of the space-charge depletion region near the grain boundaries. The redox reaction has the ability to increase the influence of space charge, evaluation of which is only possible by an electrical means. Impedance spectroscopy, $Z^{*}(f, T)$ (where $f$ is the frequency and $T$ is the temperature), could provide insights into the effects of space-charge barrier, with its ability to distinguish between the relative strengths of grain interior and grain-boundary regions, when measured in the frequency domain over a few decades of $f$. A thorough analysis with different formalisms of $Z^{*}, M^{*}, Y^{*}$, $\varepsilon^{*}$ and modeling the data to equivalent circuits facilitate the calculation of resistance $(R)$ and capacitance $(C)$ of different regions in a material. The capacitance directly implies the charge accumulation near the grain boundaries, which is thought to be a cause for the high resistivity in $\mathrm{SrTiO}_{3}$, with the resistance indicating the space-charge barrier height. There have been many reports and reviews ${ }^{14,15}$ on the impedance spectroscopy, the analysis and acquiring information using different formalisms in thick films or dense polycrystalline ceramics in contrast with very little information available on thin films. In the present work impedance spectroscopy is applied to thin films to model electrical microstructure.

Figures 4(a) and 4(b) show the complex plane plots (Col-Col plots) for the STF films deposited on alumina and sapphire at $480{ }^{\circ} \mathrm{C}$. The plots show a semicircle (a common feature observed in most polycrystalline ceramics and films attributed to one particular region) with a "spike" at low frequencies. At high frequencies the impedance ( $Z^{\prime}$ and $\left.Z^{\prime \prime}\right)$ approaches the origin indicating low impedances. The semicircle is generally modeled as a parallel combination of resistance $R$ and capacitance $C$ with its center on the real axis. With the knowledge of $R$, the capacitance $C$ can be calculated using the equation $\tau=\omega^{-1}=R C$, with $\omega=2 \pi f_{\max }$, where $\tau$ is the relaxation time, $\omega$ is the relaxation frequency, and $f_{\max }$ is the peak frequency.

The enhanced impedance response to propane is clearly reflected with an increase in impedance on the application of propane [Figs. 4(a) and 4(b)]. The resistance values calculated from the intercepts on the real axes in air and propane are in concurrence with the increase in resistance on the application of propane in the dc conductometric gas response data (Fig. 3). The higher values of resistance in films on sapphire may be due to the single-crystalline nature of its substrate, whereas alumina being polycrystalline is not prone to anisotropic effects and should partly be contributing to the overall resistance of the film.

The spike in the Col-Col plots at low frequencies indicates a simple capacitor, with an extremely small $R$ value in series with the parallel $R C$ at high frequencies of the semicircle. An inspection of the high-frequency data indicates a positive intercept on the real axis instead of a zero impedance value indicating a very low resistance value in series with the parallel $R C$ (large semicircle).

In order to accurately model the entire STF40 film, data are replotted in electrical modulus formalism, which is strongly sensitive to capacitance and low frequencies. Thus electrical modulus $\left(M^{\prime \prime}\right)$ when plotted against frequency, similar to the Bode plot of impedance, exhibits a Debye-like peak with a height which is inversely proportional to the capacitance $(C)$ of the region. By using the relaxation time $(\tau)$, the values of $R$ can be extracted (Fig. 5). 


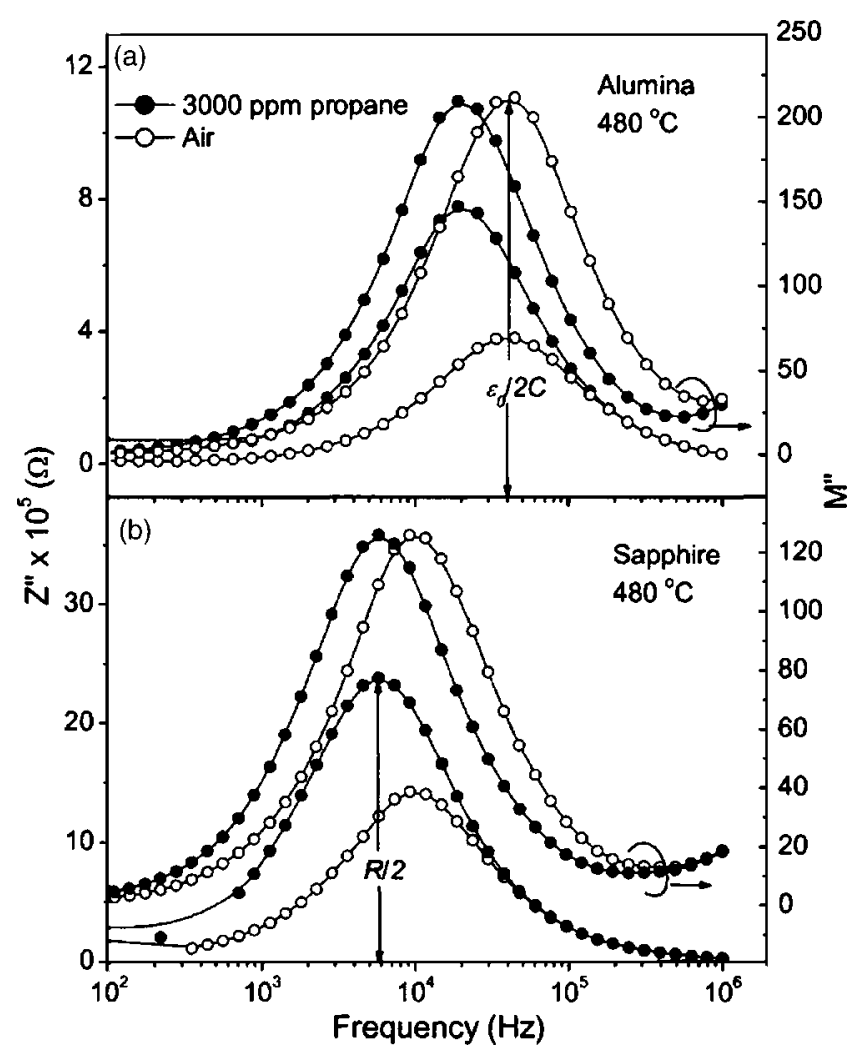

FIG. 5. Combined spectroscopic plot of $Z^{\prime \prime}$ and $M^{\prime \prime}$ for STF40 film on (a) alumina and (b) sapphire. Note that the peak height of $Z^{\prime \prime}$ is proportional to $R / 2$ and $M^{\prime \prime}$ to $\varepsilon_{0} / 2 C$. The lines are fits to the points using an equivalent circuit model.

The electrical modulus as a function of frequency is shown in Fig. 5, and exhibits Debye-like peaks around $f_{\max }$. The combined plot also indicates the $\tau$ coinciding for $Z^{\prime \prime}$ and $M^{\prime \prime}$ (solid circles or open circles in Fig. 5) at low frequencies resulting from one particular region (grain/grain boundaries) of the film. With the increase in frequency, at $f>1 \mathrm{MHz}$, the trend in $M^{\prime \prime}$ appears to show an increase resulting in an additional relaxation at frequencies higher than $1 \mathrm{MHz}$. This particularly important feature is absent in the $Z^{\prime \prime}$ spectrum, which is insensitive to capacitive effects. The combined plot of Fig. 5 thus enables us to establish and conclude that at $f$ $>1 \mathrm{MHz}$, there are additional relaxations (parallel $R C$ combination, possibly with a high capacitance) pertaining to another region of the film, in addition to the one already revealed in the complex plane. The experimental limitations did not permit obtaining a spectrum beyond the frequency of $1 \mathrm{MHz}$, which could have otherwise revealed the semicircle related to the second relaxation.

Additional information in the modulus data reveals $\left(M^{\prime \prime}\right)$ that exposure to propane induces a marginal increase in the magnitude of the $M^{\prime \prime}$ measured at $480^{\circ} \mathrm{C}$. The increase in $M^{\prime \prime}$ for the film deposited on alumina [Fig. 5(a)] is relatively greater than that of the film on sapphire [Fig. 5(b)]. This indicates an important result that additional charges are created on the exposure of propane to the film, marginally higher in the case of the film on alumina. However, the $Z^{\prime \prime}$ spectrum with a height which is directly proportional to the resistance shows a strong increase on the application of propane, with a decrease in relaxation time in both the cases.

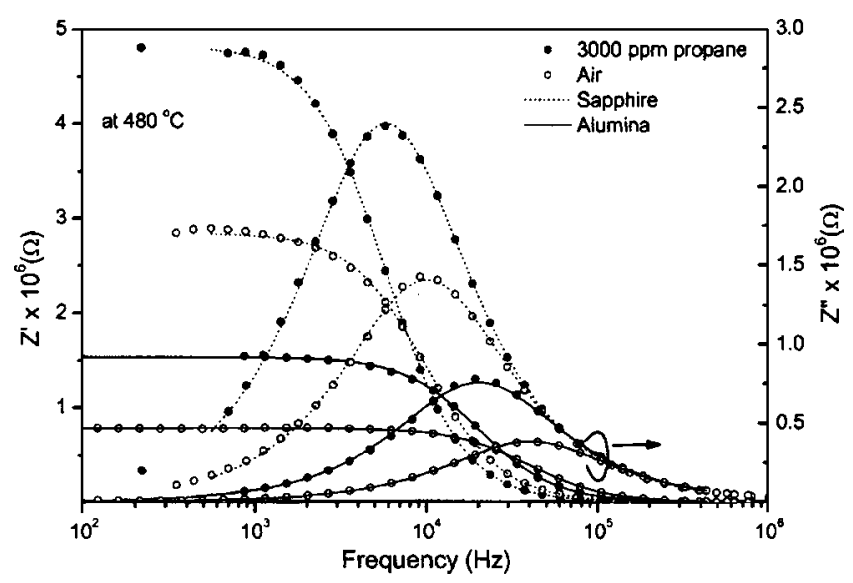

FIG. 6. Spectroscopic plots of $Z^{\prime}$ and $Z^{\prime \prime}$ for STF40 exposed to propane and air at $T=$ at $480^{\circ} \mathrm{C}$.

As revealed by the combination of impedance and modulus spectroscopy, the relatively high-frequency regime does not only contain a single region but an additional region at $f>1 \mathrm{MHz}$. A complete equivalent circuit thus consists of two parallel combinations of $R C$ s connected in series with a series capacitance (associated with the spike in Col-Col plots) at low frequencies.

Using the above analysis, a parallel combination of $R C$ can be assigned to each region. The parallel $R C$ at $f$ $>1 \mathrm{MHz}$ can be attributed to the grain interior or bulk effects. The low values of resistance observed in the Col-Col plots give credence to this assumption and also that grain interiors are generally low in resistance and offer little hindrance to mobile charges. ${ }^{11}$ The second parallel $R C$ combination, which dominates the complex impedance plane and spectroscopic plots, is due to the grain boundaries. High values of resistance are a characteristic of grain boundaries. In the ensuing sections, the reasons for high resistance near grain boundaries will be dealt with.

The grain boundaries in acceptor-doped $\mathrm{SrTiO}_{3}$ are known to generate resistances at least four orders higher than the interiors of grains. ${ }^{15}$ This is due to the presence of spacecharge depletion regions on both sides of the grain boundaries, which are generally positively charged and donor in nature. This forms a back-to-back Schottky barrier which resists the movement of charges across the grain boundaries. ${ }^{14}$ An evidence for this is obtained from the spectroscopic plots of real $\left(Z^{\prime}\right)$ and imaginary parts $\left(Z^{\prime \prime}\right)$ of the impedance, as shown in Fig. 6. In a parallel combination of $R C$ the low-frequency part of the impedance spectrum is dominated by the dc resistance until the relaxation frequency $(\tau)$ is reached. As $f \rightarrow 1 / \tau$, the reactive part of the $R C$ starts to dominate the overall impedance up to high frequencies. At low frequencies, the reactive part of the $R C$ has a high value of resistance thus effectively making dc resistance contribute to the overall impedance.

A scenario that can be visualized from the above findings is that the space-charge barrier, reflecting the $Z^{\prime \prime}$ spectra, due to the depletion of the mobile charges near the grain boundaries offers high resistance to the species attempting to cross the grain boundary. Moreover, the $Z^{\prime}$ spectrum, which is mostly due to the resistive component in $R C$ circuit, offers 


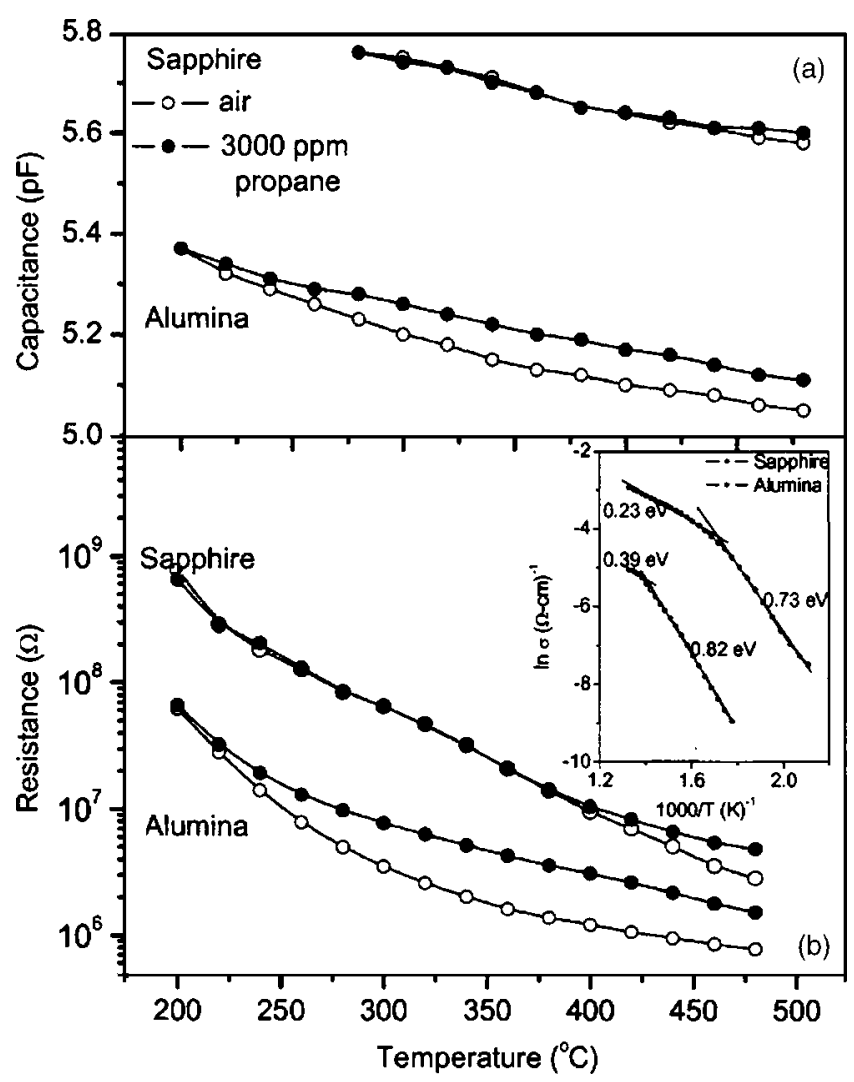

FIG. 7. (a) $C$ and (b) $R$ values calculated from the fits to the equivalent circuits as a function of temperature for the STF40 film deposited on sapphire and alumina. Inset (b) shows the dc conductivity as a function of inverse temperature. Values of activation energies $\left(E_{A}\right)$ are indicated.

relatively lower resistance and is observed up to low frequencies. At $f>1 / \tau$, the total impedance decreases as a result of the decrease in the reactance of the capacitance indicating the decline in the depletion region near the grain boundaries. The conduction mechanism at these frequencies is complex with the agitation exerted on mobile charges by the frequency resulting in charge compensation and recombination and the ionized charges moving away from the grain boundaries. The high frequencies cause a localized hopping conduction with the conduction species having insufficient time to relax. Due to this, a gas response measured at high frequencies may not yield desired results as the grain boundaries are no longer depleted of the mobile charges. This also confirms that the sensitivity of STF40 films to propane is due to the grain boundaries.

The $R$ and $C$ values as a function of temperature measured in air and propane obtained by nonlinear least-square fittings (NLSFs) are shown in Fig. 7. Films deposited on polycrystalline alumina show strong sensitivity to propane with a corresponding increase in both resistance and capacitance. Significant sensitivity in this case is obtained at temperatures as low as $275^{\circ} \mathrm{C}$ [Fig. 7(a)]. The resistance $(R)$ and corresponding space-charge depletion region capacitance $(C)$ also show a considerable increase upon exposure to propane. In contrast, the kinetics in the STF40 film deposited on sapphire does not allow any clear propane response until the temperature approaches $400{ }^{\circ} \mathrm{C}$ [Fig. 7(b)]. The significantly higher response observed in films deposited on alumina also indicates that in addition to the grain-boundary effects of the film, depletion regions with barriers for the mobile charges also are possible near the grain boundaries of the polycrystalline alumina substrate itself.

Out of several mechanisms that are in development to effectively explain the gas sensitivity, the gas diffusion model, grain-boundary model, and the open- and closed-neck models are the most important. ${ }^{16}$ The gas diffusion model uses the surface reaction and diffusion (Knudsen diffusion) of the gas into the film, to explain the material sensitivity to a gas. ${ }^{17}$ The grain-boundary model, however, uses the induced space-charge barriers as seen above near the grain boundaries, due to the reduction or oxidation in the grain interiors. $^{11}$

The inset of Fig. 7(b) shows the conductivity variation as a function of reciprocal temperature (Arrhenius plots). The conductivity increases with increasing temperature, which corresponds to the charge mobility and becomes independent of temperature as the temperature is further increased. The temperature-independent conductivity at high temperatures is a common occurrence in metal oxide semiconductors with $p$-type semiconductivity under constant oxygen partial pressures. At high temperatures, oxygen vacancies are created thermally according to

$$
\mathrm{Oo} \rightarrow V_{o}+2 e^{-}+\frac{1}{2} \mathrm{O}_{2} \text {. }
$$

In donor-doped titanates where the oxygen vacancy concentrations are low, the fine-grained ceramics sintered under oxidizing conditions show high insulating grains due to the cation vacancy occupation. ${ }^{1}$ Whereas, in an acceptor-doped $\mathrm{SrTiO}_{3}$ the increased number of oxygen vacancies introduces more defects which are compensated by the negative cation acceptor centers. This tends to decrease the conductivity. The opposing effect of thermal promotion increases the conductivity and competition leads to little change in conductivity as the temperature rises. ${ }^{1} \mathrm{~A}$ band gap of $3.2 \mathrm{eV}$ in $\mathrm{SrTiO}_{3}$ reduces to $2.1 \mathrm{eV}$ with the addition of $\mathrm{Fe}$ and increasing $\mathrm{Fe}$ doping further reduces this band gap. ${ }^{18}$ In an acceptor-doped perovskite, activation energy $\left(E_{A}\right)$ is given by ${ }^{18}$

$$
E_{A}=\left(2 E_{g}-\Delta H_{\mathrm{Red}}\right) / 2 .
$$

The band gap $\left(E_{g}\right)$ decreases with increasing impurity cation concentration and the enthalpy $\left(H_{\text {Red }}\right)$ keeps increasing with increasing temperature and oxygen vacancy concentration. Due to this, $E_{A}$ reduces to almost $0 \mathrm{eV}$ and in some cases even results in negative values at higher acceptor doping levels. ${ }^{19}$ The $E_{A}$ values at high temperatures determined above are consistent with those reported for acceptor-doped titanates. $^{18}$

The loss of oxygen at high temperature is also confirmed by TGA. The TGA in Fig. 8 shows a considerable weight loss with increasing temperature which it regains upon cooling to $450{ }^{\circ} \mathrm{C}$, although the process is not completely reversible. On heating, the weight shows an initial decrease and appears to gain at temperatures in excess of $300{ }^{\circ} \mathrm{C}$, before it loses considerable weight at $T>400{ }^{\circ} \mathrm{C}$. The initial weight loss and gain does not emanate as a consequence of the oxygen loss, and recombination could be due to extraneous effects of either moisture or other volatile contamination. The 


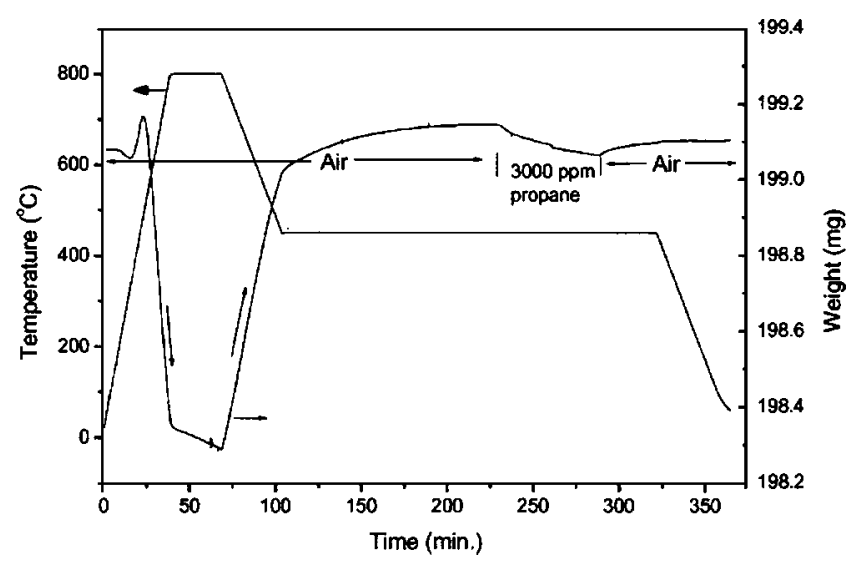

FIG. 8. Thermogravimetry of STF40, depicting the weight loss as a function of exposure to air and 3000-ppm propane.

direct effects of an oxygen loss which greatly influences the conduction mechanism were not seen in the dc conductivity plot in the temperature ranges of weight loss and gain, confirming the above assumption.

TGA at $450{ }^{\circ} \mathrm{C}$ in 3000 -ppm propane also allows insights into the mechanism which leads to an enhancement in response as a function of gas concentration. On the application of propane, a clear weight loss in the sample is observed, which is regained after its exposure to air. The weight loss is associated with the reduction reaction which can be nominally shown in the following:

$$
\begin{aligned}
& \mathrm{SrTi}_{0.6} \mathrm{Fe}_{0.4} \mathrm{O}_{3}+\mathrm{C}_{3} \mathrm{H}_{8}+(5-\delta / 2) \mathrm{O}_{2} \\
& \quad \rightarrow \mathrm{SrTi}_{0.6} \mathrm{Fe}_{0.4-\delta}^{4+} \mathrm{Fe}_{\delta}^{3+} \mathrm{O}_{3-\delta}+3 \mathrm{CO}_{2} \uparrow+4 \mathrm{H}_{2} \mathrm{O} \uparrow .
\end{aligned}
$$

Although at the molecular level the mechanism of oxidation is not completely understood, the catalytic oxidation of propane occurs with the source of oxygen both from the gas phase and from the oxygen in the STF40 lattice. This is accompanied by the redox couple of $\mathrm{Fe}^{4+} \leftrightarrows \mathrm{Fe}^{3+}$, for charge balance, which in turn contributes to the oxygen vacancies in the film.

In a model proposed recently for $p$-type semiconductors, ${ }^{20}$ the thin oxygen adsorption layer on the surface surrounding the grain-boundary layers acts as an electron trap. The electrons that are released as a result of the redox reaction diffuse towards the negative electrode and are trapped in the acceptor sites of the adsorbed oxygen near the grain boundaries. ${ }^{21}$ Due to this trapping the charge-carrier density near the grain boundaries considerably decreases and thereby increases the height of the depletion layer leading to a resistance increase. This resistance increase is consistent with the resistance rise usually observed in $p$-type semiconductors. The redox reactions are just not confined to the magnitude of depletion barrier and the trap levels created but also on the diffusion reaction and penetration of the hydrocarbon into the interiors of the film, which takes the pore sizes into consideration and does not per se deal with the substrate used.

In most ceramics, grain boundaries have smaller conductivity as compared to either the electrodes or the bulk of the region due to the depletion space-charge layers. The grainboundary thickness is directly proportional to the conductivity of the material given by ${ }^{12}$

$$
\sigma_{g b} \approx \frac{d_{g b}}{d_{b}} \sigma_{l},
$$

where $\sigma_{g b}, \sigma_{l}, d_{g b}$, and $d_{b}$ are the conductivity of the grain boundary, long-term conductivity and thickness of grain boundary, and the bulk of the material, respectively. From the above, the thickness of the grain boundary is directly proportional to its conductivity. The SEM pictures (Fig. 2) for films deposited on sapphire and alumina show varying grain-boundary thicknesses. As in most of the polycrystalline materials, the bulk conductivity is so high that it does not appear in the impedance spectrum due to the size of the grain-boundary space-charge thickness.

\section{CONCLUSIONS}

$\mathrm{SrTi}_{0.6} \mathrm{Fe}_{0.4} \mathrm{O}_{3}$ can be deposited as polycrystalline films on smooth and rough substrates. At elevated temperatures, they behave as $p$-type semiconductors and the electrical resistance increases when the films are exposed to reducing gaseous species. It was found that films substituted with $\mathrm{Fe}$ in $\mathrm{SrTiO}_{3}$ could sense hydrocarbons such as propane quite strongly and be transduced as a significant change in electrical conductivity. The magnitude of the response varies with films deposited on sapphire and alumina towards propane as a result of differences in microstructure. Analysis using impedance spectroscopy clearly reveals contribution of the grain-boundary regions to the overall resistivity of the film. The single semicircle observed in the complex plane is attributed to the grain boundaries after a thorough analysis using electrical modulus formalism, which is more sensitive to the capacitance of a particular region. Additional contributions from the grain boundaries of alumina substrate result in the higher conductivity in films deposited on alumina as compared to those deposited on single-crystal sapphire. The redox reaction induced by the presence of propane enhances the space-charge barrier across the boundaries, which is realized as sensor response. Overall, STF40 films deposited on sintered alumina appear more promising as hydrocarbon sensor than the films on sapphire.

\section{ACKNOWLEDGMENTS}

The authors thank Xiaomei Du for assistance with PLD and Jim Margeson (Institute for Research in Construction, NRC) for FEG-SEM images. Financial support of this project by National Research Council of Canada, in the NRC-Helmholtz collaborative program, is gratefully acknowledged Grant No. (NRCC-21-CRP-02).

${ }^{1}$ P. T. Moseley and A. J. Crocker, Sensor Materials (IOP, London, 1996). ${ }^{2}$ P. T. Moseley, Meas. Sci. Technol. 8, 223 (1997).

${ }^{3}$ F. H. Chibirova and E. E. Gutman, Russ. J. Phys. Chem. 74, 1555 (2000).

${ }^{4}$ D. Orlik, M. Ivanovskaya, and A. Gurlo, Zh. Anal. Khim. 52, 69 (1997).

${ }^{5}$ J. J. Tunney and M. L. Post, J. Electroceram. 5, 63 (2000).

${ }^{6}$ O. Saburi, J. Phys. Soc. Jpn. 14, 1159 (1959).

${ }^{7}$ G. D. Mahan, L. M. Levinson, and H. R. Phillipp, J. Appl. Phys. 50, 2799 (1979).

${ }^{8}$ T. Takahashi, High Conductivity Solid Ionic Conductors (World Scientific, 
Singapore, 1989).

${ }^{9}$ J. Mizusaki, M. Okayasu, S. Yamauchi, and K. Fueki, J. Solid State Chem. 99, 166 (1992).

${ }^{10}$ K. Sahner, R. Moos, M. Matam, and M. Post, Proceedings of IEEE International Conference on Sensors, Toronto, 2003 (unpublished), pp. 926931.

${ }^{11}$ R. Hagenbeck and R. Waser, J. Appl. Phys. 83, 2083 (1998).

${ }^{12} \mathrm{R}$. Waser, Ferroelectrics 151, 125 (1994).

${ }^{13}$ M. Vollmann, R. Hagenbeck, and R. Waser, J. Am. Ceram. Soc. 80, 2301 (1997).

${ }^{14}$ J. T. S. Irvine, D. C. Sinclair, and A. R. West, Adv. Mater. (Weinheim,
Ger.) 2, 132 (1990)

${ }^{15}$ J. R. Mac Donald, Impedance Spectroscopy (Wiley, New York, 1987).

${ }^{16}$ N. Barsan and U. Weimar, J. Electroceram. 7, 143 (2001).

${ }^{17}$ N. Matsunaga, G. Sakai, K. Shimanoe, and N. Yamazoe, Sens. Actuators B 83, 216 (2002).

${ }^{18}$ W. Menesklou, H.-I. Schreiner, K. H. Hardtl, and E. Ivers-Tiffee, Sens. Actuators B 59, 184 (1999).

${ }^{19}$ P. T. Moseley, Sens. Actuators B 6, 149 (1992).

${ }^{20}$ K. Sahner, R. Moos, M. Matam, J. Tunney, and M. Post, Sens. Actuators $\mathrm{B}$ (in press).

${ }^{21}$ D. M. Smyth, Ferroelectrics 151, 115 (1994). 